\title{
Astragaloside IV Inhibits the Up-Regulation of Wnt/ $\beta$-Catenin Signaling in Rats with Unilateral Ureteral Obstruction
}

\author{
Li Wang $^{\mathrm{b}}$ Yang-Feng Chi ${ }^{\mathrm{a}}$ Ze-Ting Yuanc Wen-Chao Zhou ${ }^{\mathrm{c}}$ Pei-Hao Yin ${ }^{\mathrm{a}}$ \\ Xue-Mei Zhang ${ }^{d}$ Wen Penga,b
}

aDepartment of Nephrology, 'baboratory of Renal Disease, 'Experimental Research Center, Putuo Hospital, Shanghai University of Traditional Chinese Medicine, dDepartment of Pharmacology, School of Pharmacy, Fudan University, Shanghai, China

\section{Key Words}

Astragaloside IV $\bullet$ Wnt/ $\beta$-catenin signaling pathway $\bullet$ UUO model $\cdot$ Renal interstitial fibrosis

\begin{abstract}
Objective: To investigate the effect of Astragaloside IV (AS-IV) on the regulation of the Wnt/ $\beta$ catenin signaling pathway in rats with unilateral ureteral obstruction (UUO). Methods: Rat renal interstitial fibrosis models were prepared using unilateral ureteral ligation. Rats were randomly divided into sham group, sham group with AS-IV $(33 \mathrm{mg} / \mathrm{kg})$, unilateral ureteral obstruction group, and unilateral ureteral obstruction group receiving varied doses of AS-IV $(3.3,10$, and $33 \mathrm{mg} / \mathrm{kg})$. Immunohistochemical analysis, real-time fluorescence quantitative PCR (FQ-PCR), and western blot were used to detect the expression of genes and proteins associated with the Wnt/ $\beta$-catenin signaling pathway in renal tissues. Results: Levels of Wnt3, Wnt4, and Frizzled gene expression increased significantly in the UUO model; AS-IV was associated with the downregulation of the expression of Wnt3, Wnt4, Frizzled4, p-LRP5, p-LRP6, disheveled, $\beta$-catenin, LEF-1, TCF-1, Snail, Jagged 1, Twist, MMP2, and MMP7 proteins in a concentration-dependent manner, while the expression of APC, CK1, and E-cadherin was increased. Conclusions: AS-IV effectively inhibits the up-regulation of proteins in the $\mathrm{Wnt} / \mathrm{\beta}$ catenin signaling pathway in UUO-model rats, indicating its possible inhibitory effects on renal interstitial fibrosis.
\end{abstract}




\section{Introduction}

Renal fibrosis, particularly tubulointerstitial fibrosis, is the common mediator and pathological basisfor the development of chronic kidneydiseaseand the eventual development of end-stage renal disease (ESRD) [1]. While a variety of cytokines and signaling pathways that contribute to renal fibrosis, such as TGF- $\beta / \mathrm{Smad}$, Wnt/ $\beta$-catenin, and PI3K/AKT, have been identified [2-4], the exact molecular mechanism for renal fibrosis development remains unclear. Currently there is no effective treatment available and therefore, it is necessary to discover new effective therapies for the prevention and treatment of renal fibrosis.

The Wnt/ $\beta$-catenin signaling pathway is an evolutionarily conserved pathway that regulates crucial processes including tissue and organ formation, maintenance of intracellular homeostasis, as well as disease progression (such as cancer, obesity, and diabetes)[5]. The Wnt/ $\beta$-catenin signaling pathway is maintained in a silent state in the absence of Wnt ligand, $\beta$-catenin has been identified and shown to be degraded by ubiquitin protein after its phosphorylation by the Axin/APC/CK1/GSK-3 $\beta$ composite. $\beta$-catenin expression was detected in the cytoplasm at low levels, and most of them were found in conjunction with E-cadherin, serving as cell adhesion molecules. However, in the presence of Wnt ligand, the Frizzeled (Frz) receptor dimerizes with the coreceptor, LRP5 or 6, forming a receptor/coreceptor complex. The activation of disheveled proteins (Dsh or Dvl) leads to the detachment of GSK-3 $\beta$ from the degradation complex, resulting in the inhibition of $\beta$-catenin phosphorylation and prevention of its degradation. It further leads to continuous accumulation of $\beta$-catenin in the cytoplasm and its nucleus, as well as combination with $\mathrm{T}$ cell factors (T cell factor/lymphoid enhancer factor, TCF/LEF), and subsequent activation of Snail, MMPs, twist, and Jagged1 target gene expression [5].

The Wnt/ $\beta$-catenin signaling pathway has been found to mediate kidney disease processes, such as obstructive nephropathy, diabetic nephropathy, focal glomerulosclerosis, and adriamycin nephropathy [6-9], in addition to playing a role in the pathogenesis of renal interstitial fibrosis $[10,11]$. Crosstalk between Wnt/ $\beta$-catenin, TGF- $\beta / S m a d$, and Integrin/ ILK signaling pathways at different levels has been noted as the key process in promoting epithelial to mesenchymal transition (EMT) in renal tubule cells and epithelial podocytes. This EMT transition is thought to be a direct result of renal interstitial fibrosis [12-14]. Therefore, inhibition or blockage of the Wnt/ $\beta$-catenin signaling pathway may help slow the progression of renal interstitial fibrosis.

Astragalus membranaceus is one of the most widely used traditional Chinese medicine in China for thousands of years. The chemical basis of this natural plant is actively being investigated, and its extracts are typically classified into saponins, polysaccharides and flavonoids [15]. Generally, Astragaloside IV (AS-IV) is considered as the representative active saponin compound mediating many pharmacological properties of A.membranaceus, such as anti-hypertension, positive inotropic action, anti-inflammatory and immune-regulatory effects $[16,17]$. Furthermore, there is increasing evidence that suggests AS-IV has a renal protective role via anti-inflammation, inhibiting oxidative stress and NF-kB-mediated inflammatory genes expression, attenuate podocyte injury via regulation of MAPK pathway, ameliorate potocyte apoptosis by attenuating ROS production [18-20]. In addition, there is evidence that AS-IV may also have inhibitory effects on EMT, but the specific pathways and mechanisms have not yet been fully elucidated [21].

The unilateral ureteral obstruction (UUO) model is characterized by progressive tubular atrophy and interstitial fibrosis, while the glomeruli are less affected. This model is therefore the classical model for renal interstitial fibrosis and obstructive nephropathy in rodents [22]. In previous studies where the UUO model was used, the Wnt/ $\beta$-catenin signaling pathway was activated, and most Wnt proteins and Frizzled receptors were subsequently upregulated. Additionally, $\beta$-catenin accumulation was observed in the cytoplasm and nuclei of renal tubular epithelial cells, and it induced the overexpression of target genes (MMP-7, Twist, fibronectin, and C-Myc) in the Wnt/ $\beta$-catenin pathway $[6,11]$. In the present study, the UUO model was employed to investigate the regulatory role of AS-IV in the Wnt/ $\beta$-catenin 
signaling pathway, thus providing a theoretical basis for further studies on the activity of ASIV against renal interstitial fibrosis.

\section{Materials and Methods}

\section{Drugs and reagents}

AS-IV (purity>98\%) was purchased from the Shanghai China Research Institute of Food and Drug Testing. The compound was prepared as a suspension in the medium of $0.5 \%$ carboxymethyl cellulose (CMC) for animal study. Antibodies including Phospho T1492-LRP5 (P-LRP5) and Phospho T1479-LRP6 (P-LRP6) were purchased from Abnova (Taipei City, Taiwan). Wnt4, Frizzeled4, Dishevelled, Axin, TCF-1 and Twist were purchased from Santa Cruz Biotechnology (Santa Cruz, CA, USA). Wnt3, MMP2, APC, E-cadherin, $\beta$-catenin and $\alpha$-tubulin were purchased from Epitomics (Burlingame, CA, USA). LRP5, LRP6, GSK-3 $\beta$, CK1, Non-Phospho (Active) $\beta$-catenin (Ser33/37/Thr41), $\beta$-actin, Snail, Jagged 1, MMP7 and goat anti-rabbit IgGHRP were provided by Cell Signaling Technology (Danvers, MA, USA). LEF-1 was purchased from Prosci (California, MO, USA) and Histone 2B from Abcam (Cambridge, MA, USA). Enhanced chemiluminescence system (ECL) plus was purchased from Millipore (Billerica, MA, USA). A cytoplasm nucleus extraction kit was purchased from Cayman (Michigan, MO, USA) and a BCA protein assay kit from Pierce (Rockford, IL, USA). Primers were synthesized from Sangon (Shanghai Co. Ltd., China).

\section{Animal model and grouping}

All experimental procedures were approved by the ethics committee of Putuo Hospital, Shanghai University of Traditional Chinese Medicine and complied with the National Animal Safety Specifications. Male Sprague-Dawley rats (age: 8 weeks; weight, 180-220 g) were provided by the Shanghai SLAC Experimental Animal Co. Experimental animals were randomly divided into six groups, each containing 20 animals, as follows: the sham group, the sham group with AS-IV (33 mg/kg), the UUO group, and the UUO group with varied doses of AS-IV $(3.3,10$, and $33 \mathrm{mg} / \mathrm{kg})$. AS-IV was given to rats by oral gavage the day before the experiment began and continued for 14 days. Meanwhile, $0.5 \%$ CMC was given to the sham group or the UUO group. Rats were anesthetized with 3\% chloral hydrate by intraperitoneal injection. Skin incisions were made, and the fascia and muscle were dissected to expose the kidney structures. The left ureter was first ligated at its entry of the renal hilum and a second ligation was made more distal to the hilum. The ureter was cut between the two ligations to prevent retrograde infection. The operation was completed by suturing the muscle, subcutaneous tissue, and skin. Non-ligated ureters were dissected from the sham group only.

\section{Specimen collection and preparation}

The rats were sacrificed 14 days after surgery. Gross observations of bilateral renal tissues were performed to assess the changes. The obstructed kidneys were carefully removed and sagittally sliced into two parts. The first part was snap frozen in liquid nitrogen and kept at $-80^{\circ} \mathrm{C}$ for protein and RNA extraction, and the second part was immersed into $10 \%$ neutral formalin for immunohistochemical staining.

\section{Immunohistochemistry of the renal tissue}

Immunohistochemistry was performed using a two-step kit (Vector Laboratories, Burlingame, CA, USA) according to protocols specified in the kit instructions. DAB coloring and mild hematoxylin staining were done prior to microscopic observation of Wnt 3 and Wnt 4 expression in the cortical tubular interstitial area. Positive signals were defined as the nucleus and the cytoplasm appearing as brown particles under the fluorescence microscope (Leica, Germany). Semi-quantitative analysis was performed using Image Pro Plus 6.0 Multimedia Color Pathological image analysis software (Media Cybernetics, Inc. Silver Spring, MD, USA). Five non-overlapping high-power fields were selected in the cortical areas under light microscope. A total of 500 cells were counted in each field, avoiding the glomeruli and blood vessels. The mean optical density value of Wnt 3 and Wnt 4 were calculated. Expression of $\beta$-catenin in the nucleus was observed, and the percentage of positive cell units was calculated. 
Table 1. Primers used for quantitative Real-time PCR

\begin{tabular}{lll}
\hline Genes & \multicolumn{1}{c}{ Forward Primer } & \multicolumn{1}{c}{ Reverse Primer } \\
\hline Wnt1 & 5'- TGCAAGTGGCAATTCCGAAAC-3' & 5'-AGCCTCGGTTGACGATCTTG-3' \\
Wnt2 & 5'-TGGACAGCTGCGAAGTTATGT-3' & 5'-AGTTGTCCAGTCGGCACTC-3' \\
Wnt2b & 5'-CACCTTCCTCCACCCTCAATC-3' & 5'-ATCAGGGAGCCACTCAGTCTC-3' \\
Wnt3 & 5'-GCACAGGGAAGGGGTAGATT-3' & 5'-AGAGCAGGGTGAGAGACGTT-3' \\
Wnt4 & 5'-GTTCCACACTGGACTCCCTG-3' & 5'-ACACCTGCTGAAGAGATGGC-3' \\
Wnt5a & 5'-GCTTTGCCAAGGAGTTCGTG-3' & 5'-AGGTTCATGAGGATGCGAGC-3' \\
Wnt6 & 5'-CTGACTCGCCACTGGTCTT-3' & 5'-TACTTTTCCTAGGGAGCTGCTG-3' \\
LRP1 & 5'-CCCAGAGATGCCCACCAAAT-3' & 5'-GTAGGGACGCAATGGTGCT-3' \\
LRP2 & 5'-ACTGCACTACCCTGTGTTCG-3' & 5'-TGCGCTCATCGCTGTTATCT-3' \\
LRP3 & 5'-TGGAGCTACGACTGGGTTATG-3' & 5'-GTTGCTGCGGTAGGAAAGC-3' \\
LRP4 & 5'-GTGCTGAGGAGGGATACTGC-3' & 5'-ATAACCGGCTTCGCACCAG-3' \\
LRP5 & 5'-ACTGGAGGGCTACGTGTACT-3' & 5'-AGGCGAGTCACCTCAATTCTG-3' \\
LRP6 & 5'-AGACTACGACCCCGTGGAAG-3' & 5'-CAATGAAGGAGCGACGGATG-3' \\
3-actin & 5'-GCAGGAGTACGATGAGTCCG-3' & 5'-ACGCAGCTCAGTAACAGTCC-3' \\
\hline & &
\end{tabular}

Western blot analysis

For total tissue protein, frozen renal samples were lysed in a buffer (50 mM Tris at PH of 7.5, 1 mM EDTA, $150 \mathrm{mM} \mathrm{NaCl}, 20 \mathrm{mM} \mathrm{NaF}, 0.5 \%$ NP-40, 10\% glycerol, 1\% protease inhibitor cocktail and 1\% phosphatase inhibitor cocktail) by sonication on ice. The lysates were cleared by centrifugation at 14,000 $\mathrm{g}$ at $4^{\circ} \mathrm{C}$ for $20 \mathrm{~min}$ and the supernatant was collected. For cytoplasmic and nuclear protein, samples were lysed according to cytoplasmic and nuclear extraction kit. Briefly, the samples were lysed in ice-cold 1× hypotonic buffer supplemented with dithiothreitol and $10 \%$ NP-40 by sonication on ice. Thereafter, the samples were incubated on ice for $15 \mathrm{~min}$ and then centrifuged at $14,000 \mathrm{~g}$ at $4^{\circ} \mathrm{C}$ for $30 \mathrm{~s}$. The supernatant which contains cytoplasmic protein was collected. Then the cell pellets containing nuclei were retained and resuspended in ice-cold $1 \times$ extraction buffer with protease and phosphatase inhibitors. The samples were incubated on a shaker platform for $10 \mathrm{~min}$ followed by centrifugation at $14,000 \mathrm{~g}$ at $4^{\circ} \mathrm{C}$ for $10 \mathrm{~min}$. The supernatant contains the nuclear fraction. Then the protein concentration was determined by BCA protein assay kit. Equal amounts of protein $(20 \mu \mathrm{g})$ were separated by $10 \%$ SDS polyacrylamide gel electrophoresis and transferred to PVDF (Millipore, MO, USA) membrane. Following blocked with 5\% BSA in TBS-Tween-20 for $2 \mathrm{~h}$, the membrane was incubated with primary antibodies individually with Wnt3 (1:5000 dilution), Wnt4 (1:100 dilution), Frizzled4 (1:200 dilution), LRP5 (1:500 dilution), p-LRP5 (1:1000 dilution), LRP6 (1:500 dilution), p-LRP6 (1:1000 dilution), dishevelled (1:1000 dilution), GSK-3 $\beta$ (1:1000 dilution), Axin (1:200 dilution), APC (1:250 dilution), CK1 (1:1000 dilution), $\beta$-catenin (1:1000 dilution), Active $\beta$-catenin (1:10,000 dilution), LEF-1 (1:1000 dilution), TCF-1 (1:200 dilution), Snail (1:500 dilution), Jagged1 (1:500 dilution), E-cadherin (1:500 dilution), Twist (1:200 dilution), MMP-2 (1:1000 dilution), MMP-7 (1:1000 dilution), $\beta$-actin (1:1000 dilution), Histone $2 \mathrm{~B}\left(1: 1000\right.$ dilution) and $\beta$-tubulin $(1: 1000$ dilution $)$ at $4{ }^{\circ} \mathrm{C}$ overnight. After three washes in TTBS, the membranes were incubated with Horseradish peroxidase (HRP)labeled goat anti-rabbit IgG for $1 \mathrm{~h}$ at room temperature. Protein bands were visualized using enhanced ECL and X-ray film. The ratio of the protein interested was subjected to $\beta$-actin, Histone $2 \mathrm{~B}$ or $\alpha$-tubulin and was densitometrically analyzed by Multi-Analyst software program (Version 1.01; Bio-Rad).

\section{Real-time fluorescence quantitative PCR (FQ-PCR)}

Total RNA was extracted from frozen renal samples using the RNeasy Mini Kit (QIAGEN, Valencia, CA) following the manufacturer's protocol. Briefly, $1 \mu \mathrm{g}$ of total RNA was reverse transcribed using the AMV First Strand cDNA Synthesis kit (QIAGEN, Valencia, CA) and synthesized complementary DNA was amplified by a standard PCR protocol using SYBR green PCR master mix (QIAGEN, Valencia, CA). Primers were synthesized from Sangon Biotech Co. Ltd. (Shanghai, China). The sequences of rat-specific primers for Wnt 1, 2, 2b, 3, 4, 5a, 6, LRP 1, 2, 3, 4, 5, 6 and $\beta$-actin used in the study were enlisted in Table 1 . Cycling conditions were: 15 min preincubation at $95^{\circ} \mathrm{C}, 10 \mathrm{sec}$ denaturation at $95^{\circ} \mathrm{C}, 31 \mathrm{sec}$ annealing at $58^{\circ} \mathrm{C}$ for 40 cycles using $\mathrm{ABI}$ PRISM 7300 sequence detection system (Applied Biosystems). In order to confirm amplification specificity, the PCR products from each primer pair were subjected to a melting curve analysis. Each reaction was amplified in triplicate and the threshold cycles $(\mathrm{Ct})$ were calculated using the $2^{-\Delta \Delta \mathrm{Ct}}$ method. Relative gene expression was normalized with $\beta$-actin as an internal reference. 
Fig. 1. Wht and sFRP gene expression detected by FQ-PCR: A and B. Relative mRNA levels of different subtypes of Wnt and sFRP gene expression in sham and UUO group. Each column represents the mean \pm SEM obtained from 10 to 12 samples. ${ }^{*} \mathrm{P}<0.05$, compared with the sham group.

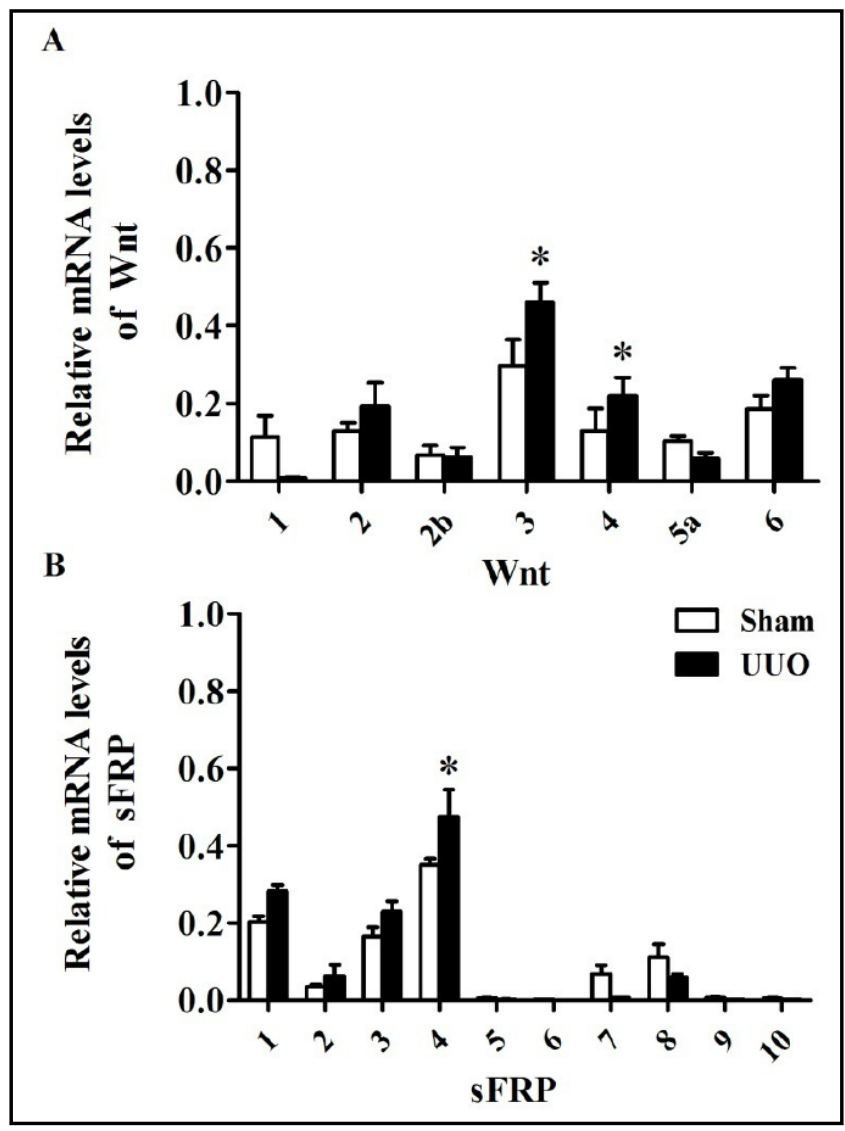

Statistical analysis

GraphPad Prism 5.0 software (GraphPad Prism software Inc., San Diego, Calif, USA) was used for statistical analysis. One-way ANOVA and Student's $t$-test were used for two-group and multi-group comparisons. Data are presented as the mean \pm SEM. P-value of less than 0.05 was considered statistically significant.

\section{Results}

AS-IV inhibited membrane-level expression of Wnt/ $\beta$-catenin signaling pathway proteins

Systematic analysis of the mRNA expression of Wnt genes and Frizzled genes (sFRP) were first detected in both the sham and UUO groups in the absence of AS-IV. As shown in Fig. 1, Wnt3, Wnt4, and sFRP4 expression levels increased significantly in the UUO group when compared to the sham group $\left({ }^{*} \mathrm{P}<0.05\right)$. Next, the AS-IV efficacy on protein level expression of Wnt3, Wnt4, and Frizzled4 during the course of renal interstitial fibrosis induced by UUO was further investigated. As shown in Fig. 2, the three protein levels in the UUO group were significantly increased when compared to the sham group ( $\left.{ }^{* * *} \mathrm{P}<0.001\right)$, while the expression

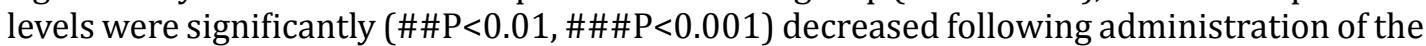
different doses of AS-IV (3.3, 10, $33 \mathrm{mg} / \mathrm{kg})$. A similar trend was also detected for P-LRP5 and P-LRP6 expression (Fig. 2).

Immunohistochemical analysis demonstrated a dose-dependent reduction of Wnt3 and Wnt4 expression with AS-IV administration in the UUO model rats (Fig. 3).

AS-IV inhibited cytoplasmic-level expression of Wnt/ $\beta$-catenin signaling pathway proteins

The levels of disheveled protein, $\beta$-catenin, and active $\beta$-catenin decreased as the concentrations of AS-IV increased, while the expression levels of APC and CK1 tended to increase as the AS-IV dosage increased among the cytoplasmic proteins ( $\# \mathrm{P}<0.05, \# \# \mathrm{P}<0.01)$ (Fig. 4). 
A

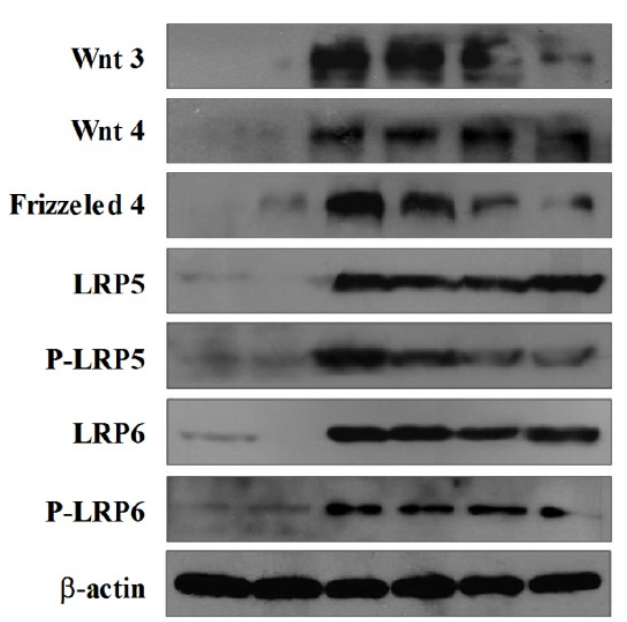

C

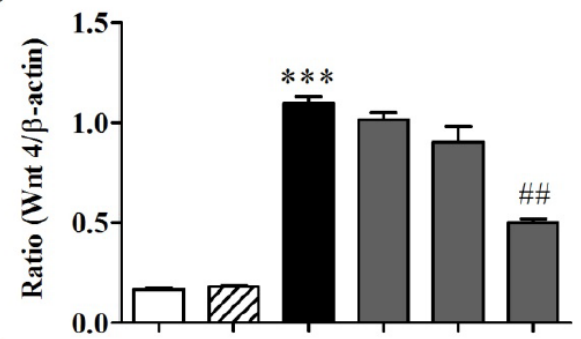

$\mathbf{E}$

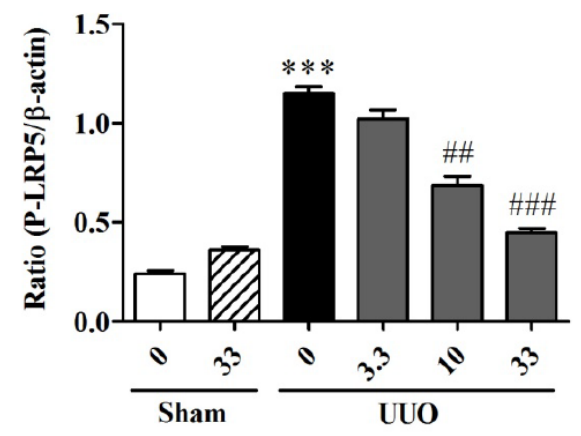

B

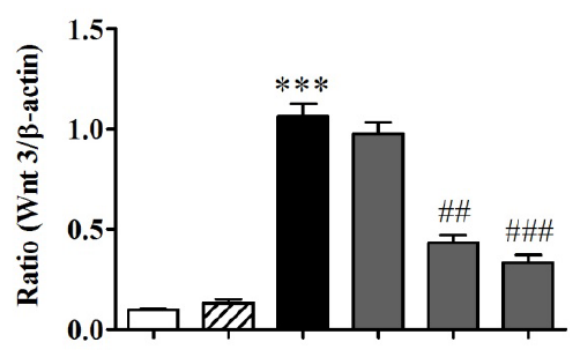

D

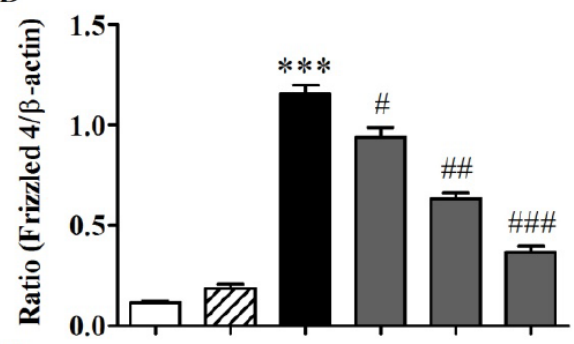

F

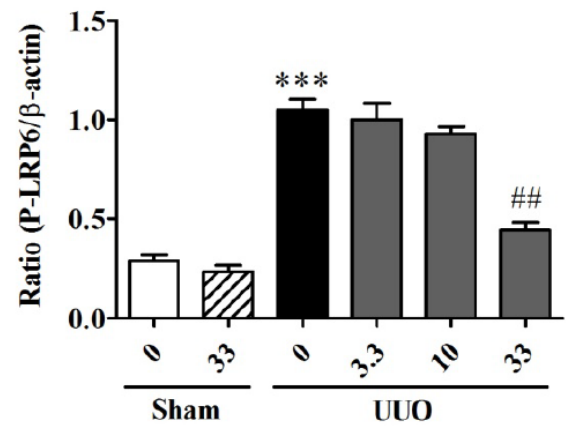

Fig. 2. Effects of AS-IV on the expression of Wnt/ $\beta$-catenin signaling pathway membrane proteins: A. Representative western blot photographs showing levels of Wnt3, Wnt4, Frizzled4, LRP 5,6, and P-LRP 5,6 protein expressions in kidneys; B-F. Quantitative analysis of the relative ratios of Wnt3, Wnt4, Frizzled4, and P-LRP 5,6 protein normalized with $\beta$-actin. Data represent mean \pm SEM for at least 3 independent experiments. ${ }^{*} \mathrm{P}<0.05$, compared with the sham group; $\# \mathrm{P}<0.05$, \#\#P<0.01, \#\#\#P<0.001, compared with the UUO group.

Further observation of active $\beta$-catenin expression in the nucleus by immunohistochemistry showed a significant increase in active $\beta$-catenin expression in the UUO model $\left({ }^{* * *} \mathrm{P}<0.001\right)$, and it was shown that AS-IV could reverse this trend in a concentrationdependent manner (\#\#P<0.01, \#\#\#P<0.001) (Fig 5. A, B). The same trend was observed for nuclear protein expression (Fig 5. C).

\section{AS-IV inhibited nuclear-level expression of Wnt/ $\beta$-catenin signaling pathway proteins}

The expression of LEF-1, TCF-1, Snail, Jagged, E-cadherin, Twist, MMP2, and MMP7 proteins was detected among the total proteins. The levels of all proteins, except for E-cadherin, were found elevated in the UUO model. Different concentrations of AS-IV reduced the expression of such proteins $(\# \mathrm{P}<0.05$, \#\#P<0.01, \#\#\#P<0.001) (Fig. 6). 


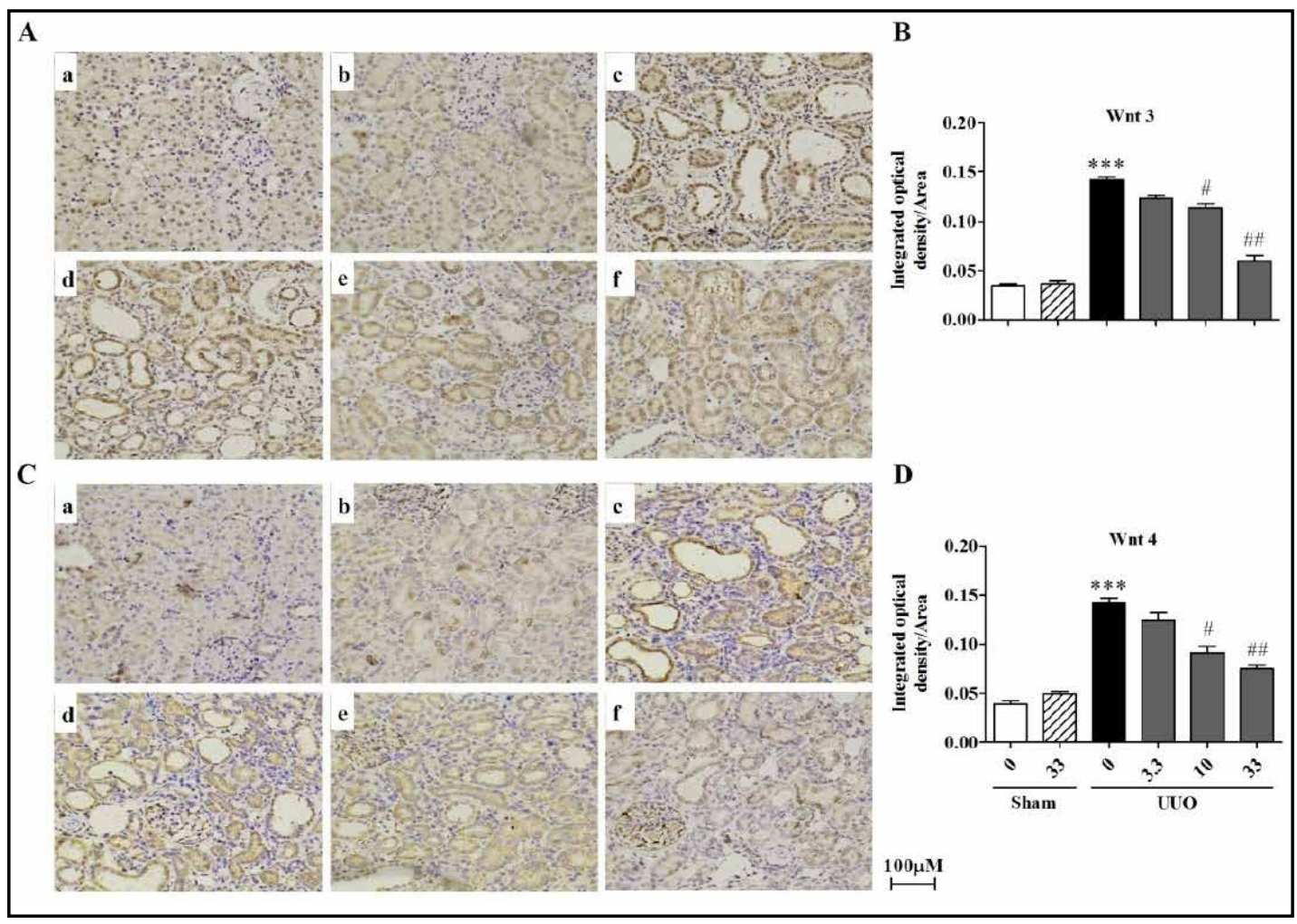

Fig. 3. Effects of AS-IV on Wnt3 and Wnt4 expression in UUO model rats: A and C. Representative photomicrographs of Wnt3 and Wnt4 immunohistochemistry on kidney sections from the sham (a), sham group with AS-IV $33 \mathrm{mg} / \mathrm{kg}$ (b), UUO (c), UUO group with AS-IV 3.3, 10, and $33 \mathrm{mg} / \mathrm{kg}$ (d-f) groups. B and D. Average integrated optical density (integrated optical density/area) analysis of Wnt3 and Wnt4 expression from sections similar to those shown in A and C. Each column represents the mean \pm SEM obtained from 12 to 15 samples. ${ }^{*} \mathrm{P}<0.05$, compared with the sham group; $\# \mathrm{P}<0.05$, \#\#P<0.01, compared with the UUO group. Magnification: $\times 200$.

\section{Discussion}

In the present study, we found that the AS-IV monomer, used in Chinese medicine, inhibited the activation of the Wnt/ $\beta$-catenin signaling pathway in UUO model rats in a concentration-dependent manner. This effect was associated with altered expression of multiple proteins at various levels, including membrane receptors and ligands, cytoplasmic, and nuclear proteins. These results suggest that AS-IV may have a preventive effect on renal interstitial fibrosis by inhibiting the Wnt/ $\beta$-catenin signaling pathway.

Wnt is a glycoproteinsecreted in an autocrine or paracrine fashion. Wnt signaling is divided into three mechanisms according to the ligands and their associated receptors: the classical Wnt/ $\beta$-catenin pathway (canonical Wnt $/ \beta$-catenin), the planar cell polarity pathway, and the $\mathrm{Wnt} / \mathrm{Ca}^{2+}$ pathway [23]. The classical $\mathrm{Wnt} / \beta$-catenin pathway is composed mainly of the tetramer of Wnt ligands (Wnt1, 3, 8, and 8b), Frz, and LRP5/6. The signal would be transmitted to Dsh proteins within the cytoplasm and inhibit the GSK-3 $\beta$, thus contributing to the accumulation of $\beta$-catenin in the cytoplasm. The planar cell polarity pathway is activated by Frz and non-canonical Wnt ligands (Wnt4, 5, and 11). The Dsh expression also needs to be activated, thereby causing downstream JHK or RhoA activation. The Wnt/Ca ${ }^{2+}$ pathway can increase intracellular calcium levels via G proteins [24]. Although Wnt4 is also able to activate the non-canonical Wnt signaling pathway, in this experiment, we believe that the regulatory role of AS-IV for Wnt4 still contributes to the inhibition of Wnt/ $\beta$-catenin signaling pathway. 


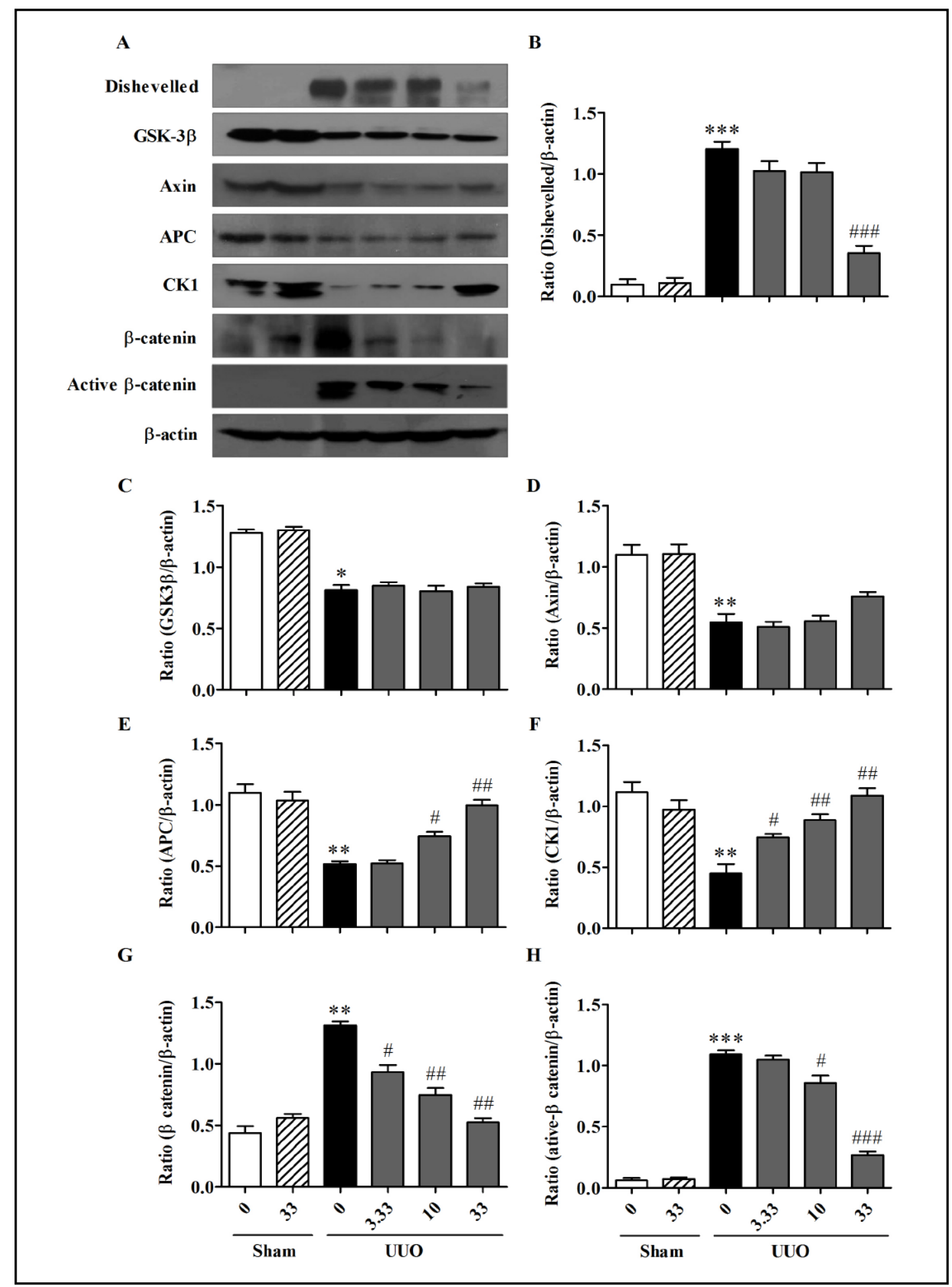

Fig. 4. Effects of AS-IV on the expression of induced Wnt/ $\beta$-catenin signaling pathway cytosolic proteins in the UUO model: A. Representative western blot photographs showing levels of Disheveled, GSK-3 $\beta$, Axin, APC, CK1, $\beta$-catenin, and Active $\beta$-catenin protein expressions in kidneys; B-H. Summary histograms showing the ratio of Disheveled, GSK-3 $\beta$, Axin, APC, CK1, $\beta$-catenin, and Active $\beta$-catenin expressions relative to $\beta$-actin from experiments similar to those shown in A. Data represent mean \pm SEM for at least 3 independent experiments. ${ }^{*} \mathrm{P}<0.05$, ${ }^{* *} \mathrm{P}<0.01$, ${ }^{* * *} \mathrm{P}<0.001$, compared with the sham group; \#P<0.05, $\# \# \mathrm{P}<0.01$,\#\#\#P<0.001, compared with the UUO group. 
A
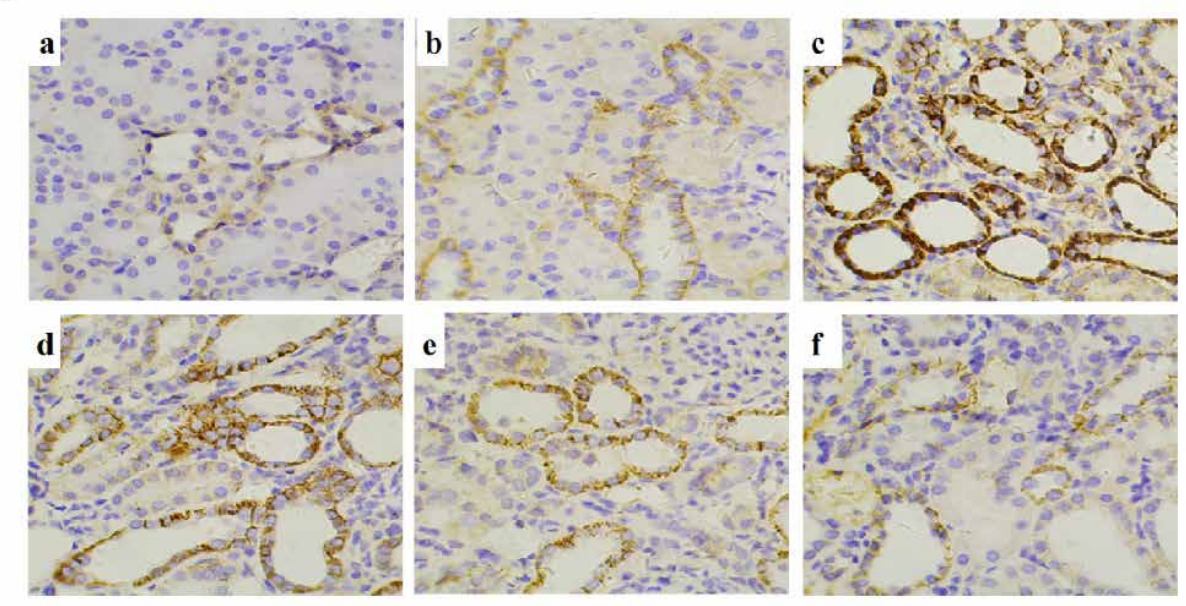

B

C
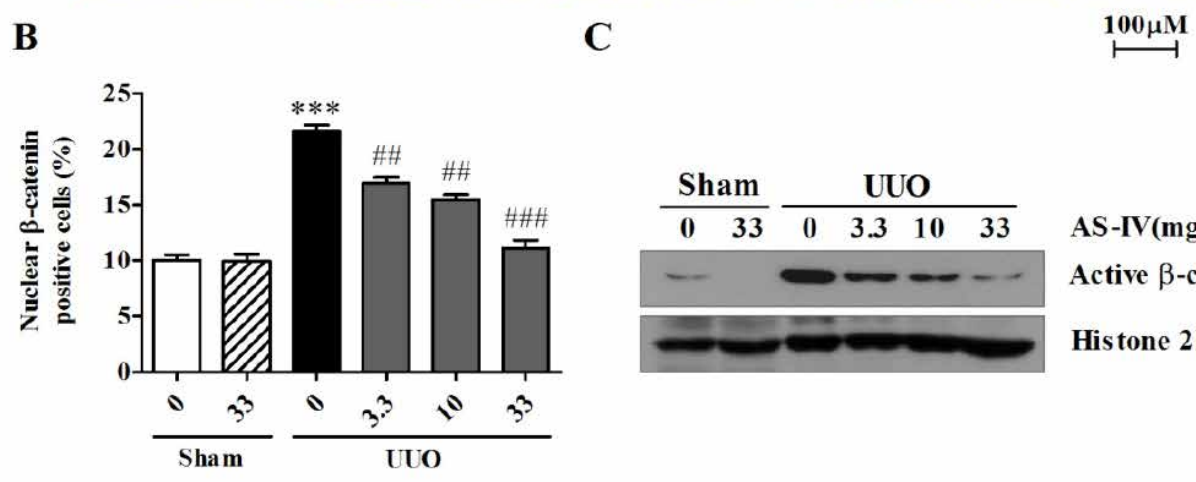

$\operatorname{AS}-I V(\mathbf{m g} / \mathbf{k g})$

Active $\beta$-catenin

His tone 2B

Fig. 5. Effects of AS-IV on nuclear $\beta$-catenin expression caused by the UUO model: A. Representative photomicrographs of nuclear $\beta$-catenin immunohistochemistry on kidney sections from the sham (a), sham group with AS-IV $33 \mathrm{mg} / \mathrm{kg}$ (b), UUO (c), UUO group with AS-IV 3.3, 10, and $33 \mathrm{mg} / \mathrm{kg}$ (d-f) groups. B. Nuclear $\beta$-catenin positive cells (\%) analysis from sections similar to those shown in A. Each column represents the mean \pm SEM obtained from 12 to 15 samples. C. Representative western blot photographs showing levels of active $\beta$-catenin expression in kidneys; ${ }^{* * *} \mathrm{P}<0.001$, compared with the sham group; \#\#P<0.01, $\# \# \#$ P $<0.001$, compared with the UUO group. Magnification: $\times 400$.

First, AS-IV was shown to inhibit Wnt4 expression in a concentration-dependent manner (Fig. 3). This result is consistent with previous reports suggesting that in obstructive renal tissue, fibrosis usually occurs around the collecting tube along with high expression of Wnt 4 , and that Wnt 4 also induced $\beta$-catenin in fibroblasts cultured in vitro to travel into the nucleus $[11,25-27]$. Secondly, $\beta$-catenin, the key factor in the Wnt/ $\beta$-catenin pathway, in the cytoplasm or in the nucleus, was inhibited by AS-IV (Fig. 4 and 5). The expression of $\beta$-catenin increased during renal fibrosis, suggesting that it may be one of the key points for targeted therapy. Lastly, MMP-7, designated as the "biological identity" in the UUO model rats representing the activity of Wnt/ $\beta$-catenin pathway, was found to be associated with Wnt4 expression in renal tubular injury $[28,29]$. The results confirmed the finding that ASIV can significantly reduce MMP-7 expression (Fig. 6).

EMT is an important mechanism in the development of tubulointerstitial fibrosis. EMT not only increases the presence of interstitial fibroblasts and extracellular matrix (ECM) deposition, but also aggravates tubular epithelial damage that is characterized by the reduced or absent expression of epithelial cell E-cadherin and the increased expression of plasminogen activator inhibitor-1 (PAI1), Snail, and MMPs within fibroblasts [30]. E-cadherin molecules work in the cytoskeleton to maintain the structure and polarity in 
A

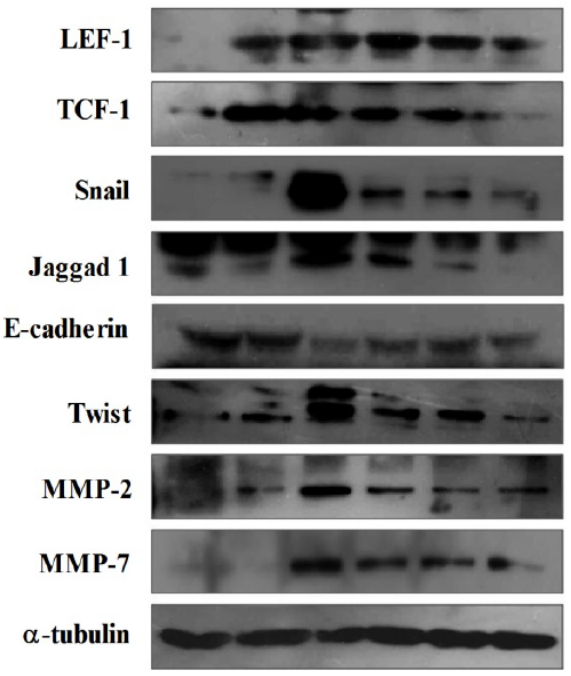

D

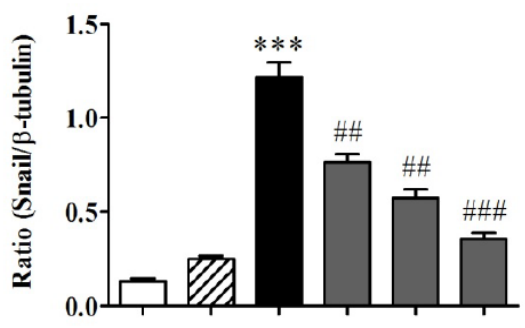

F

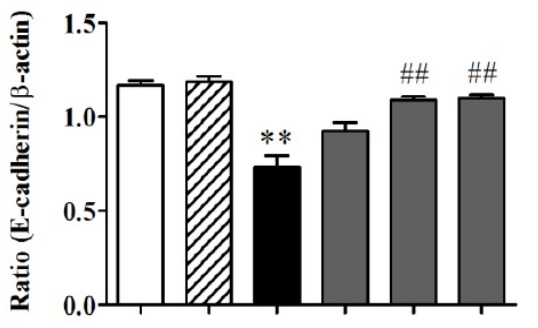

H

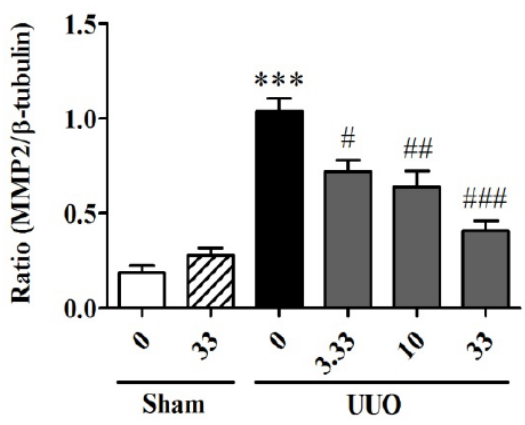

B

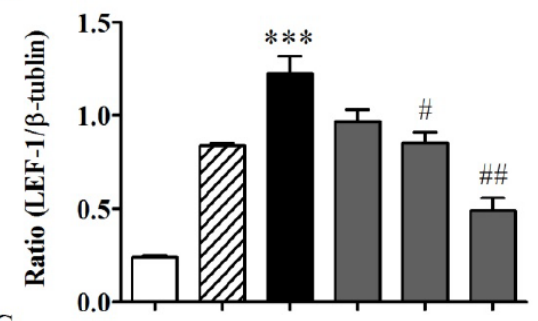

C

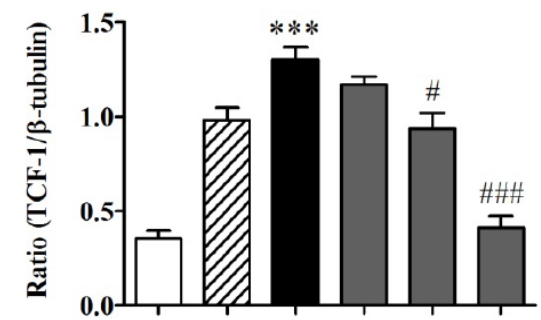

E

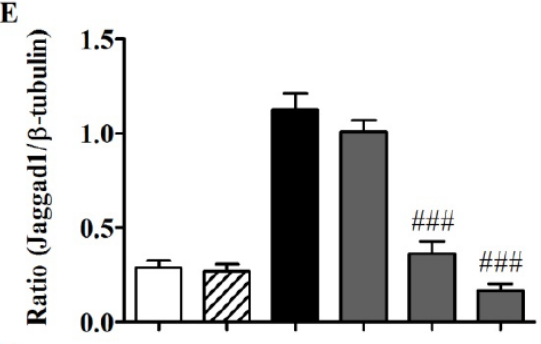

G
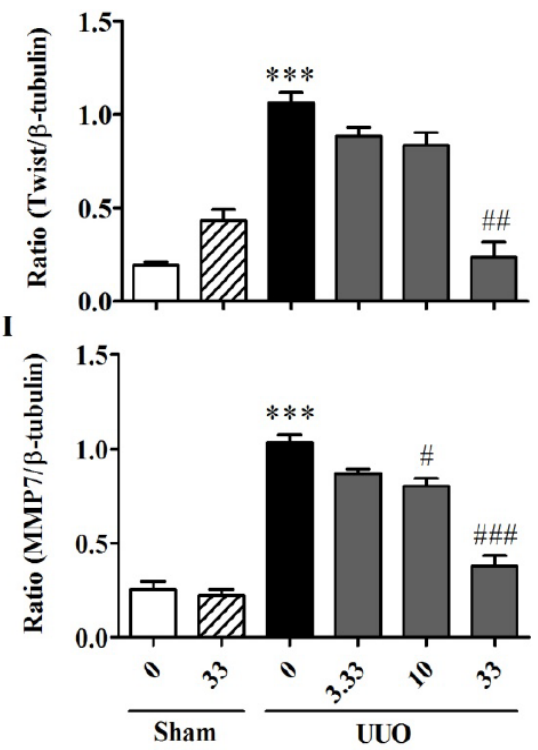

Fig. 6. Effects of AS-IV on the expression of downstream transcription factors of the Wnt/ $\beta$-catenin signaling pathway in the UUO model: A. Representative western blot photographs showing levels of LEF-1, TCF-1, Snail, Jagged, E-cadherin, Twist, MMP2, and MMP7 protein expressions; B-I. Quantitative analysis of the relative ratios of LEF-1, TCF-1, Snail, Jagged, E-cadherin, Twist, MMP2, and MMP7 protein normalized with $\alpha$-tubulin. Data represent mean \pm SEM for at least 3 independent experiments. ${ }^{* * *} \mathrm{P}<0.001$, compared with the sham group; \# $\mathrm{P}<0.05$, \#\#P<0.01, \#\#\#P<0.001, compared to the UUO group. 
various cells, including renal tubular epithelial cells. The expression of E-cadherin can also inhibit $\beta$-nuclear transcription [31]. MMPs could promote EMT progress by direct inhibition of E-cadherin expression [32-35]. Interestingly, most of the target genes in the Wnt/ $\beta$ catenin signaling pathway, e.g., Snail, Twist, LEF1, and Jagged1, could also work as regulators or transcription factors of EMT. Snail overexpression inhibits the expression of E-cadherin in renal tubular epithelial cells, and consequently induces EMT in transgenic mice [36, 37]. Twist cannot only inhibit E-cadherin, but also up-regulate the expression of mesenchymal markers such as fibronectin, $\alpha$-SMA, and vimentin [38]. Our experiments revealed that ASIV has a regulatory role in the expression of most of the target genes of the Wnt/ $\beta$-catenin signaling pathway, as well as factors of EMT (Fig. 6), suggesting that AS-IV may be involved in the EMT process by inhibiting the Wnt/ $\beta$-catenin signaling pathway.

The Wnt/ $\beta$-catenin signaling pathway is tightly regulated through intracellular proteinprotein interactions. Since the Wnt/ $\beta$-catenin signaling pathway is involved in many disease processes, a variety of antagonistic endogenous molecules have been recently found. One particular series of antagonists binds to Wnt proteins, while the other binds to the Wnt receptor complex [39]. Because of the limited effects of these antagonists, several researchers are turning their focus towards the development of new formulations to restore normal Wnt/ $\beta$-catenin activity. Studies have found that paricalcitol, a structural analogue of vitamins, for example, could inhibit the $\mathrm{Wnt} / \beta$-catenin pathway and alleviate the damage to podocyte function, thus alleviating proteinuria and renal damage [40]. The development of therapeutic regimens from natural products is a novel and important directionin biomedical research.

In the present study, we found that the inhibitory activity of AS-IV to Wnt/ $\beta$-catenin signaling could not only regulate the levels of Wnt and its receptors, but also change the expression of APC, CK1, and and TCF/LEF to varying degrees (Fig. 4 and 6). Commonly, those proteins can affect the activity of $\beta$-catenin through change the binding capability, so one reason for the protein changes may due to the reduction of $\beta$-catenin binding to those factors in the pathway. However, previous study also demonstrates that LEF- 1 can induce EMT directly when its transcription activity is activated by stable nuclear $\beta$-catenin, and APC can control the nuclear accumulation of $\beta$-catenin by a combination of nuclear export and cytoplasmic degradation mechanism $[41,42]$. Therefore, we consider that the role of those proteins may not just bind to $\beta$-catenin, which in turn affect the proportion of nuclear $\beta$-catenin positive cells. In other words, those changes themselves may regulate the process of AS-IV inhibits the up-regulation of Wnt/ $\beta$-catenin signaling, and more details need to be studied in the future.

It is noteworthy that AS-IV is not completely inhibitory of Wnt/ $\beta$-catenin signaling in the UUO model. This is partly explained by the fact that the Wnt $/ \beta$-catenin signaling pathway is not the only pathway that AS-IV could act on [43]. Moreover, factors such as the modeling time of the UUO mice, the route of administration, and the dosage may also affect the activity of the drug [44].

In summary, the results of our present study demonstrated the inhibitory effect of ASIV on the Wnt/ $\beta$-catenin signaling pathway in rat UUO model, and further suggested that AS-IV may affect renal interstitial fibrosis through this pathway, indicating that AS-IV may potentially act as a renal protective agent.

\section{Acknowledgements}

This work was supported by General Medicine of Key Discipline Construction Project, State Administration of Traditional Chinese Medicine of the People's Republic of China; Leading Academic Discipline Project of State Administration of Traditional Chinese Medicine of China, Talent Project of Integrative Medicine of Shanghai Municipal Health Bureau (ZYSNXD012-RC-ZXY); Key Medical Discipline Project of Shanghai Municipal Health Bureau (ZK2012A34); Independent Innovation Research Fund of Putuo District Science 
and Technology Committee (2012PTKW002) and Budget Project of Shanghai Municipal Education Commission (2012JW71).

\section{Disclosure Statement}

No.

\section{References}

1 Zeisberg M, Neilson EG: Mechanisms of tubulointerstitial fibrosis. J Am Soc Nephrol 2010;21:1819-1834.

-2 Ning XH, Ge XF, Cui Y, An HX: Ulinastatin inhibits unilateral ureteral obstruction-induced renal interstitial fibrosis in rats via transforming growth factor beta (TGF-beta)/Smad signalling pathways. Int Immunopharmacol 2013;15:406-413.

- 3 Ho C, Lee PH, Hsu YC, Wang FS, Huang YT, Lin CL: Sustained Wnt/beta-catenin signaling rescues high glucose induction of transforming growth factor-beta1-mediated renal fibrosis. Am J Med Sci 2012;344:374-382.

-4 Qin J, Xie YY, Huang L, Yuan QJ, Mei WJ, Yuan XN, Hu GY, Cheng GJ, Tao LJ, Peng ZZ: Fluorofenidone inhibits nicotinamide adeninedinucleotide phosphate oxidase via PI3K/Akt pathway in the pathogenesis of renal interstitial fibrosis. Nephrology 2013;18:690-699.

5 MacDonald BT, Tamai K, He X: Wnt/beta-catenin signaling: components, mechanisms, and diseases. Dev Cell 2009:17:9-26.

-6 Surendran K, Schiavi S, Hruska KA: Wnt-dependent beta-catenin signaling is activated after unilateral ureteral obstruction, and recombinant secreted frizzled-related protein 4 alters the progression of renal fibrosis. J Am Soc Nephrol 2005:16:2373-2384.

7 Dai C, Stolz DB, Kiss LP, Monga SP, Holzman LB, Liu Y: Wnt/beta-catenin signaling promotes podocyte dysfunction and albuminuria. J Am Soc Nephrol 2009:20:1997-2008.

8 Naves MA, Requião-Moura LR, Soares MF, Silva-Júnior JA: Mastroianni-Kirsztajn G, Teixeira VP, Podocyte Wnt/ss-catenin pathway is activated by integrin-linked kinase in clinical and experimental focal segmental glomerulosclerosis. J Nephrol 2012;3:401-409.

-9 He W, Tan RJ, Li Y, Wang D, Nie J, Hou FF, Liu Y: Matrix metalloproteinase-7 as a surrogate marker predicts renal Wnt/beta-catenin activity in CKD. J Am Soc Nephrol 2012;23:294-304.

10 Guo Y, Xiao L, Sun L, Liu F: Wnt/beta-catenin signaling: a promising new target for fibrosis diseases. Physiol Res 2012;61:337-346.

11 He W, Dai C, Li Y, Zeng G, Monga SP, Liu Y: Wnt/beta-catenin signaling promotes renal interstitial fibrosis. J Am Soc Nephrol 2009;20:765-776.

12 Liu Y: New insights into epithelial-mesenchymal transition in kidney fibrosis. J Am Soc Nephrol 2010;21:212-222.

13 He J, Xu Y, Koya D, Kanasaki K: Role of the endothelial-to-mesenchymal transition in renal fibrosis of chronic kidney disease. Clin Exp Nephrol 2013;17:488-497.

14 Li R, Wang Y, Liu Y, Chen Q, Fu W, Wang H, Cai H, Peng W, Zhang X: Curcumin inhibits transforming growth factor-beta1-induced EMT via PPARgamma pathway, not Smad pathway in renal tubular epithelial cells. PLoS One, 2013;8:e58848.

15 Yu QT, Qi LW, Li P, Yi L, Zhao J, Bi Z: Determination of seventeen main flavonoids and saponins in the medicinal plant Huang-qi (Radix astragali) by HPLC-DAD-ELSD. J Sep Sci 2007;30:1292-1299.

16 Qiu LH, Xie XJ, Zhang BQ: Astragaloside IV improves homocysteine-induced acute phase endothelial dysfunction via antioxidation. Biol Pharm Bull 2010:33:641-646.

17 Zhao J, Yang P, Li F, Tao L, Ding H, Rui Y, Cao Z, Zhang W: Therapeutic effects of Astragaloside IV on myocardial injuries: multi-target identification and network analysis. PLoS One 2012;7:e44938.

18 Gui D, Huang J, Liu W, Guo Y, Xiao W, Wang N: Astragaloside IV prevents acute kidney injury in two rodent models by inhibiting oxidative stress and apoptosis pathways. Apoptosis 2013;18:409-22.

19 Gui D, Guo Y, Wang F, Liu W, Chen J, Chen Y, Huang J, Wang N: Astragaloside IV, a novel antioxidant, prevents glucose-induced podocyte apoptosis in vitro and in vivo. PLoS One 2013; 7:e39824.

20 Gui D, Huang J, Guo Y, Chen J, Chen Y, Xiao W, Liu X, Wang N: Astragaloside IV ameliorates renal injury in streptozotocin-induced diabetic rats through inhibiting NF-kappaB-mediated inflammatory genes expression.Cytokine 2013;61:970-977. 


\begin{tabular}{|c|c|c|}
\hline Cellular Physiology & Cell Physiol Biochem 2014;33:1316-1328 & \\
\hline and Biochemistry & $\begin{array}{l}\text { DOI: } 10.1159 / 000358699 \\
\text { Published oniline: April Z8, } 2014\end{array}$ & $\begin{array}{l}\text { O 2014 S. Karger AG, Basel } \\
\text { www.karger.com/cpb }\end{array}$ \\
\hline
\end{tabular}

-21 Qi W, Niu J, Qin Q, Qiao Z, Gu Y: Astragaloside IV attenuates glycated albumin-induced epithelialto-mesenchymal transition by inhibiting oxidative stress in renal proximal tubular cells. Cell Stress Chaperones 2014;19:105-114.

-22 Chevalier RL, Forbes MS, Thornhill BA: Ureteral obstruction as a model of renal interstitial fibrosis and obstructive nephropathy. Kidney Int 2009;75:1145-1152.

23 Komiya Y, Habas R: Wnt signal transduction pathways. Organogenesis 2008;4:68-75.

24 Filipovich A, Gehrke I, Poll-Wolbeck SJ, Kreuzer KA: Physiological inhibitors of Wnt signaling. Eur J Haematol 2011;86:453-465.

25 Michael DG, Nusse R: Wnt signaling: multiple pathways, multiple receptors, and multiple transcription factors. J Biol Chem 2006;11:22429-22433.

-26 Gordon MD, Nusse R: Wnt4/beta-catenin signaling in medullary kidney myofibroblasts. J Am Soc Nephrol 2013;24:1399-1412.

27 Surendran K, McCaul SP, Simon TC: A role for Wnt-4 in renal fibrosis. Am J Physiol Renal Physiol 2002;282:F431-F441.

28 He W, Tan RJ, Li Y, Wang D, Nie J, Hou FF, Liu Y: Matrix metalloproteinase-7 as a surrogate marker predicts renal Wnt/beta-catenin activity in CKD. J Am Soc Nephrol 2012;23:294-304.

29 Surendran K, Simon TC, Liapis H, McGuire JK: Matrilysin (MMP-7) expression in renal tubular damage: association with Wnt4. Kidney Int 2004;65:2212-2222.

-30 Kalluri R, Neilson EG: Epithelial-mesenchymal transition and its implications for fibrosis. J Clin Invest 2003;112:1776-1784.

-31 Orsulic S, Huber O, Aberle H, Arnold S, Kemler R: E-cadherin binding prevents beta-catenin nuclear localization and beta-catenin/LEF-1-mediated transactivation. J Cell Sci 1999;112:1237-1245.

32 Zheng G, Lyons JG, Tan TK, Wang Y, Hsu TT, Min D, Succar L, Rangan GK, Hu M, Henderson BR, Alexander SI, Harris DC: Disruption of E-cadherin by matrix metalloproteinase directly mediates epithelial-mesenchymal transition downstream of transforming growth factor-beta1 in renal tubular epithelial cells. Am J Pathol 2009;175:580-591.

-33 Du X, Shimizu A, Masuda Y, Kuwahara N, Arai T, Kataoka M, Uchiyama M, Kaneko T, Akimoto T, Iino Y, Fukuda Y: Involvement of matrix metalloproteinase-2 in the development of renal interstitial fibrosis in mouse obstructive nephropathy. Lab Invest, 2012;92:1149-1160.

34 Tan TK, Zheng G, Hsu TT, Wang Y, Lee VW, Tian X, Wang Y, Cao Q Wang Y, Harris DC: Macrophage matrix metalloproteinase-9 mediates epithelial-mesenchymal transition in vitro in murine renal tubular cells. Am J Pathol 2010;176:1256-1270.

-35 Zhao H, Dong Y, Tian X, Tan TK, Liu Z, Zhao Y, Zhang Y, Harris DCh, Zheng G: Matrix metalloproteinases contribute to kidney fibrosis in chronic kidney diseases. World J Nephrol 2013;2:84-89.

-36 Tan X, Li Y, Liu Y: Paricalcitol attenuates renal interstitial fibrosis in obstructive nephropathy. J Am Soc Nephrol 2006;17:3382-3393.

37 Boutet A, De Frutos CA, Maxwell PH, Mayol MJ, Romero J, Nieto MA: Snail activation disrupts tissue homeostasis and induces fibrosis in the adult kidney. EMBO J 2006;25:5603-5613.

-38 Yang MH, Wu KJ: TWIST activation by hypoxia inducible factor-1 (HIF-1): implications in metastasis and development. Cell Cycle 2008;7:2090-2096.

39 Kawano Y, Kypta R: Secreted antagonists of the Wnt signalling pathway. J Cell Sci 2003;116:2627-2634.

40 He W, Kang YS, Dai C, Liu Y: Blockade of Wnt/beta-catenin signaling by paricalcitol ameliorates proteinuria and kidney injury. J Am Soc Nephrol 2011;22:90-103.

41 Kim K, Lu Z, Hay ED: Direct evidence for a role of beta-catenin/LEF-1 signaling pathway in induction of EMT. Cell Biol Int 2002;26:463-476.

42 Henderson BR: Nuclear-cytoplasmic shuttling of APC regulates beta-catenin subcellular localization and turnover. Nat Cell Biol 2000;2:653-660.

43 Meng LQ Tang JW, Wang Y, Zhao JR, Shang MY, Zhang M, Liu SY, Qu L, Cai SQ, Li XM: Astragaloside IV synergizes with ferulic acid to inhibit renal tubulointerstitial fibrosis in rats with obstructive nephropathy. Br J Pharmacol 2011;162:1805-1818.

44 Chevalier RL, Forbes MS, Thornhill BA: Ureteral obstruction as a model of renal interstitial fibrosis and obstructive nephropathy. Kidney Int 2009;75:1145-1152. 